\title{
La elisión de /d/ en posición intervocálica en la comunidad caraqueña ${ }^{1}$
}

\author{
Irania Malaver ${ }^{2}$ \\ Lorena Perdomo \\ Universidad Central de Venezuela
}

\begin{abstract}
Resumen
En esta comunicación se estudia la /d/ en posición intervocálica en la comunidad caraqueña en el marco del proyecto PRESEEA (Proyecto para el estudio sociolingüístico del español de España y América). Se trata de una investigación sociolingüística en la que se exploran los factores lingüísticos y extralingüísticos que influyen en el debilitamiento y elisión de la /d/ intervocálica en posición interna de palabra en el corpus PRESEEA Caracas 2004-2010. Para el análisis acústico de 3648 casos se empleó el Praat (Boersma y Weenink 2009), y el GoldVarb X (Sankoff et al. 2005) para el análisis de regresión. Los resultados muestran un 58\% de elisión en la comunidad caraqueña; en cuanto a las variables lingüísticas que inciden se deben destacar las variables de entorno vocálico y de difusión léxica. Las variables sociales muestran poca incidencia en el fenómeno.
\end{abstract}

Palabras clave: sociolingüística, cambio lingüístico, fonema /d/.

\footnotetext{
1 Proyecto "Estudio sociolingüístico de la /d/ intervocálica en el español de Venezuela" adscrito al Instituto de Filología "Andrés Bello", UCV con el código PIIFAB2010.

$2 \quad$ Para correspondencia, dirigirse a: Irania Malaver (irania.malaver@ucv.ve) o Lorena Perdomo (lorenavpp@gmail.com), Instituto de Filología "Andrés Bello" (IFAB), Facultad de Humanidades y Educación, Universidad Central de Venezuela, Edificio de la Biblioteca Central, Piso nº 11 , Los Chaguaramos, 1080 Caracas, Venezuela.
} 


\title{
The Elision of / D / INTERVOCALIC IN THE CARACAS COMMUnity
}

\begin{abstract}
In this research the intervocalic /d/ is studied in the linguistic community of Caracas as part of the project PRESEEA (Project for the Socio-linguistic Study Spanish in Spain and America). This is a sociolinguistic research which analyses the linguistic and extralinguistic factors influencing the weakening and elision of the /d/ internal position word in the corpus PRESEEA Caracas 2004-2010. For the acoustic analysis of 3648 cases, it was used Praat (Boersma and Weenink 2009), and GoldVarb X (Sankoff et al 2005) for the regression analysis. The results shows $58 \%$ of elision in the Caracas community. In as for the linguistic variables that influence should be highlighted the vocalic environment variables and the lexical diffusion. The social variables show little impact on the phenomenon.
\end{abstract}

Keywords: sociolinguistics, linguistic change, phoneme / d /.

Recibido: 30/03/2016

Aceptado: 06/07/2016

\section{INTRODUCCIÓN}

El debilitamiento y elisión del fonema/d/ (dado/dao $)$ es un proceso extendido en el español peninsular y americano: "De los cambios fónicos nacidos en los ss. XVI-XVIII los de mayor capacidad expansiva han sido el yeísmo y la desaparición de la -d- intervocálica (en cambio, el seseo o la aspiración y pérdida de -s- han permanecido en sus zonas originarias)" (Cano Aguilar 1992: 261). Moreno Fernández destaca que el proceso de [ợ] $\rightarrow\left[{ }^{\mathrm{d}}\right] \rightarrow$ $[\varnothing]$ es de origen interno y está presente en la mayoría de las comunidades dialectales; en su trabajo, este autor caracteriza las comunidades en tres grupos de acuerdo al grado del debilitamiento que exponen: (A) dialectos con predominio de la dental fricativa plena; (B) dialectos con predominio de la dental relajada; y (C) dialectos con predominio de la elisión (Moreno Fernández 2004: 100). En el caso venezolano, las investigaciones realizadas en la década de los 70 por D' Introno y Sosa $(1979,1986)$, mostraron que en Caracas la retención era bastante más frecuente que la elisión, por lo que Moreno Fernández adscribe la comunidad caraqueña al grupo (A); también mostraron que la elisión de /d/ es propia de hombres con bajo grado de instrucción, rasgo constante en las comunidades en las que el segmento /d/ 
ha sido estudiado, como bien ha planteado Moreno Fernández: "es digna de comentario la coincidencia del perfil del hablante en que más se elide la dental, prácticamente en todo el mundo hispánico: se trata de hombres, de edades avanzadas, de origen rural y de extracción social o niveles socioculturales bajos" (2004: 101).

Nuestro estudio tiene dos objetivos: el primero es describir las realizaciones plena, relajada y elidida de /d/ intervocálica en el corpus caraqueño y el segundo es examinar las variables lingüísticas y sociales que inciden en la alternancia de las variantes plena y elidida en la comunidad de habla caraqueña.

Este trabajo se estructura en cuatro partes; a la presente introducción le sigue el primer apartado dedicado a los estudios sobre la /d/ intervocálica realizados con muestras sociolingüísticas de dos ciudades del área centronorte del país (Caracas y Puerto Cabello). En la segunda parte se expone la metodología de la investigación; en la tercera se presentan los resultados y la discusión. La última sesión corresponde a las conclusiones generales.

\section{LA -/D/- EN EL ESPAÑOL VENEZOLANO}

La -/d/- en el español de Venezuela ha sido estudiada por D'Introno y Sosa $(1979,1986)$ y Navarro $(1983,1995)$, quienes definieron objetivos y metodologías similares al analizar solo el contexto intervocálico en posición interna de palabra; ambos estudios representan una contribución esencial a la sociolingüística de la /d/ en el español americano. Otros trabajos de corte sociolingüístico son los de Díaz-Campos y Gradoville (2011) y Díaz-Campos, Gradoville y Fafulas (2011). En el primer estudio, los autores se plantean un estudio comparativo (con datos de distintos dialectos americanos) acerca del papel de la difusión léxica en dos fenómenos lingüísticos, uno de ellos la elisión de /d/ intervocálica, tanto en posición interna como al principio de palabra (te digo, desde, casa de); en el segundo trabajo, Díaz-Campos, Gradoville y Fafulas (2011) analizan la incidencia de la edad y la clase social sobre la retención y elisión de -/d/-. En lo que sigue se exponen únicamente los resultados de los trabajos de D'Introno y Sosa (1979) y de Navarro (1995) pues comparten metodologías similares con nuestro estudio ${ }^{3}$.

\footnotetext{
3 Otra investigación sobre /d/ fue hecha por Márquez, Rojas y Villamizar (2006) basados en lectura de palabras con un corpus de tres niños. Dado que analizamos en este trabajo los
} 
Con un corpus de la década de los 70, D'Introno y Sosa (1979) analizaron 6077 casos de /d/ en el habla caraqueña ${ }^{4}$. Para la descripción de las realizaciones del fonema los autores definieron tres variantes, a saber: dental oclusiva, transición casi vocálica y elidida ${ }^{5}$. Hay que llamar la atención acerca de la definición de la variante de transición, pues aceptarla o no tiene consecuencias en la interpretación del fenómeno de la elisión. Esta variante es un sonido de corta duración no consonántico: "La transición es, para un oído no acostumbrado, difícil de apreciar y, desde el punto de vista articulatorio, difícil de caracterizar", afirman los autores (1979: 35), quienes advierten que, desde el punto de vista acústico, esta variante no es un segmento, aunque es sociolingüísticamente significativa, pues mientras la elisión es una variante estigmatizada, la transición no lo es.

Las variables independientes del estudio fueron el contexto fonético y la categoría gramatical. El contexto fonético se restringió a /d/ en posición interna de palabra, precedida por vocal tónica y seguida por las vocales [a] $\mathrm{u}[\mathrm{o}]$, y la categoría gramatical tomó en cuenta solo adjetivos y nombres. También se consideraron las variables sexo, nivel socioeconómico. Al concluir el análisis, se tiene que la variante dental obtuvo el $68 \%$ y las variantes de transición y elisión obtuvieron el $21 \%$ y $12 \%$, respectivamente 6 . Los contextos (tabla 1) que se muestran más favorables a la elisión son -ado, con el 19\%, y -oda, con el 10\%; por el contrario, -uda resultó favorecer la retención.

mismos entornos de D'Introno y Sosa (1979) y Navarro (1995), y que los datos de Díaz-Campos y Gradoville (2011) incluyen otro contexto fónico, no consideramos posible comparar nuestros resultados con el estudio de estos últimos dos autores. Vale acotar que la comparación con D'Introno y Sosa y Navarro tampoco es del todo exacta debido a los entornos y contextos gramaticales que decidieron analizar estos autores; no obstante, sus resultados se pueden contrastar con los datos del PRESEEA-Caracas.

4 Tomados de 36 entrevistas pertenecientes a hablantes caraqueños, 18 hombres y 18 mujeres entre 30 y 45 años, de tres niveles socioeconómicos (alto, medio y bajo).

5 La dental, a su vez, se subdividía en las variantes oclusiva, fricativa y relajada.

6 Ya que el español venezolano forma parte de la zona dialectal caribeña, cabía esperar un porcentaje de elisión mayor, en consonancia con las tendencias de otros dialectos caribeños estudiados en la misma sincronía: 23\% en el habla de República Dominicana (Alba 1999, 2000), 20\% en Panamá (Cedergren 1973). Alba (1999, 2000) propuso que el porcentaje de elisión mostrado por la comunidad caraqueña podía deberse al hecho de considerar la transición como una variante separada de la variable elidida; en Puerto Rico, López Morales (1983) reporta $21 \%$ de elisiones y señala "estos datos [Caracas] no resultan del todo comparables pues bajo "dentales" se han agrupado las fricativas plenas como las relajadas y se ha hecho categoría aparte con la transición” (1983: 125). 
Tabla 1. Porcentajes de uso de las variantes por contexto fonológico (D'Introno y Sosa 1986: 148)

\begin{tabular}{|c|c|c|c|c|}
\hline Contextos & $\mathrm{N}$ & \% Dental & \% Transición & \% Elisión \\
\hline$-a d o$ & 2163 & 52 & 29 & 19 \\
\hline- oda & 362 & 65 & 25 & 10 \\
\hline- odo & 907 & 71 & 21 & 9 \\
\hline$-a d a$ & 904 & 75 & 19 & 6 \\
\hline$-e d a$ & 81 & 93 & 2 & 5 \\
\hline- -edo & 111 & 89 & 6 & 5 \\
\hline$-i d o$ & 782 & 81 & 15 & 4 \\
\hline$-u d o$ & 215 & 90 & 6 & 4 \\
\hline$-i d a$ & 528 & 89 & 8 & 3 \\
\hline$-u d a$ & 163 & 99 & 1 & -- \\
\hline
\end{tabular}

Atendiendo a la variable categoría gramatical, los resultados (tabla 2) muestran que los adjetivos (adjetivos y participios pasados) favorecen la variante elidida con $14 \%$ y los nombres, la dental, con $6 \%$.

Tabla 2. Porcentajes de uso de las variantes por categoría gramatical

(D’Introno y Sosa 1986: 152)

\begin{tabular}{|l|c|c|}
\hline Variantes & $\%$ Nombres & \% Adjetivos \\
\hline Dental & 80 & 61 \\
\hline Transición & 15 & 24 \\
\hline Elisión & 6 & 14 \\
\hline
\end{tabular}

Según el análisis del nivel socioeconómico ${ }^{7}$, los caraqueños del nivel alto emplean la dental en un $73 \%$ y los del nivel bajo, la elidida en un $59 \%$. Como señalan D'Introno y Sosa, las diferencias significativas se dan entre los porcentajes de la dental y de la elidida, puesto que las diferencias porcentuales de uso de la variante de transición entre los niveles socioeconómicos resultaron mínimas. 
Tabla 3. Número de ocurrencias y porcentajes de uso de las variantes por nivel socioeconómico

(D'Introno y Sosa 1979:40)

\begin{tabular}{|l|c|c|c|c|c|c|c|c|}
\cline { 2 - 9 } \multicolumn{1}{c|}{} & \multicolumn{2}{c|}{ Alto } & \multicolumn{2}{c|}{ Medio } & \multicolumn{2}{c|}{ Bajo } & \multicolumn{2}{c|}{ Total } \\
\cline { 2 - 10 } \multicolumn{1}{c|}{} & $\mathrm{N}$ & $\%$ & $\mathrm{~N}$ & $\%$ & $\mathrm{~N}$ & $\%$ & $\mathrm{~N}$ & $\%$ \\
\hline Dental & 1527 & 73 & 1505 & 70 & 1091 & 59 & 4123 & 68 \\
\hline Transición & 394 & 19 & 436 & 20 & 422 & 23 & 1252 & 21 \\
\hline Elisión & 162 & 8 & 208 & 10 & 332 & 18 & 702 & 12 \\
\hline Total & 2083 & 34 & 2149 & 35 & 1845 & 30 & 6077 & 100 \\
\hline$\chi^{2}=137.42>9,49$ & $=0,000<0,05$ \\
\hline
\end{tabular}

Los valores de la $\chi^{2}$ y de $\mathrm{p}$ confirman la relación entre el debilitamiento y elisión de -/d/- con el grado de instrucción. D’Introno y Sosa confirman con estos datos que la/d/ es un marcador social entre los caraqueños y proponen que: “(...) las variantes que corresponden a los dos extremos de la escala de debilitamiento de la /d/ son las que reciben una función y un valor social más definido e importante" (1979: 41). En cuanto al sexo, los autores encontraron que la elisión es más frecuente entre los hombres (13\%) que entre las mujeres (10\%).

Navarro (1995), en su estudio de la comunidad de Puerto Cabello ${ }^{8}$, examinó 1654 casos de /d/ intervocálica pertenecientes a 65 hablantes (seleccionados según la edad, el sexo y el grado de instrucción); el autor encontró un $72 \%$ de retenciones y un $28 \%$ de elisiones (tabla 4 ), un porcentaje bastante más elevado que el reportado para Caracas por D'Introno y Sosa $(1979)^{9}$. En un segundo análisis, Navarro examinó solo los contextos en los que la vocal tónica precede la consonante (tabla 5), el participio y sus terminaciones (tabla 6) y la palabra todo (tabla 7$)^{10}$.

\footnotetext{
8 Puerto Cabello es una ciudad ubicada en el centro-norte del país, perteneciente a la misma zona dialectal de Caracas.

9 En Navarro 1983, el autor analizó 1209 casos solo del entorno vocal tónica_vocal átona, el mismo contexto analizado por D'Introno y Sosa. Para esos datos, la elisión fúe del 36\%.

10 El autor excluyó el contexto del diptongo decreciente (estudio, fastidio o media) pues inhibe la elisión de forma categórica, al igual que los casos como todavía, en los que la consonante aparece entre átonas. La decisión de analizar todo de forma independiente se basó en que era la única lexía en la que aparecían los entornos -ódo y -óda.
} 
Tabla 4. Frecuencias de las variantes del fonema -/d/- en el habla de Puerto Cabello (Navarro 1995: 136)

\begin{tabular}{|l|c|c|}
\hline Variantes & $\mathrm{N}$ & $\%$ \\
\hline Retención & 1183 & 72 \\
\hline Elisión & 471 & 28 \\
\hline Total & 1654 & 100 \\
\hline
\end{tabular}

Tabla 5. Realización de las variantes del fonema /d/ en V́\#V

(Basado en Navarro 1995: 139-40)

\begin{tabular}{|l|c|c|c|c|c|c|}
\cline { 2 - 7 } \multicolumn{1}{c|}{} & \multicolumn{2}{c|}{ Retención } & \multicolumn{2}{c|}{ Elisión } & \multicolumn{2}{c|}{ Total } \\
\cline { 2 - 7 } \multicolumn{1}{c|}{} & $\mathrm{N}$ & $\%$ & $\mathrm{~N}$ & $\%$ & $\mathrm{~N}$ & $\%$ \\
\hline -ádo & 170 & 47 & 190 & 53 & 360 & 47 \\
\hline -áda & 150 & 84 & 28 & 16 & 178 & 23 \\
\hline -éde & 19 & 66 & 10 & 34 & 29 & 4 \\
\hline -édo & 12 & 80 & 3 & 20 & 15 & 2 \\
\hline -ído & 89 & 70 & 39 & 30 & 128 & 17 \\
\hline -ída & 48 & 79 & 13 & 21 & 61 & 8 \\
\hline Total & 488 & 63 & 283 & 37 & 771 & 100 \\
\hline
\end{tabular}

Lo primero que debe destacarse es el incremento de la elisión en el entorno restringido, que pasa de $28 \%$ a $37 \%$ de los casos. La combinación -ádo es el contexto más frecuente y posee un $53 \%$ de casos de elisión; el segundo entorno más frecuente fue -áda pero este frena claramente la pérdida del segmento (16\%). Los contextos -ído y -éde tienen 39\% y 34\% de elisión, respectivamente. También la vocal $i$ frena la elisión pues la variante plena predomina en los entornos ido e $i d a^{11}$, con el $70 \%$ y $79 \%$, respectivamente. En relación con la categoría gramatical cuando -ádo es terminación no participial, la elisión se da en un 58\%, un porcentaje notablemente superior al que manifiestan los otros contextos (tabla 6).

11 La elisión en el contexto fónico -áda se concentra, principalmente, en los casos del vocablo nada (Navarro 1995). 
Tabla 6. Elisión y retención -/d/- en los contextos -ádo, -ido, -ída y -áda, en formas participiales y no participiales (Navarro 1995: 141-43) ${ }^{12}$

\begin{tabular}{|c|c|c|c|c|c|}
\hline & \multirow{2}{*}{ Contextos } & \multicolumn{2}{|c|}{ Retención } & \multicolumn{2}{|c|}{ Elisión } \\
\hline & & $\mathrm{N}$ & $\%$ & $\mathrm{~N}$ & $\%$ \\
\hline \multirow{4}{*}{ Formas no participiales } & -ádo & 61 & 42 & 83 & 58 \\
\hline & $-a ́ d a$ & 100 & 83 & 20 & 17 \\
\hline & -ído & 24 & 92 & 2 & 8 \\
\hline & $-i d a$ & 36 & 78 & 10 & 22 \\
\hline \multicolumn{6}{|c|}{$\chi^{2}=62.81>7.815$ y de $p=0,000<0,05$} \\
\hline \multirow{4}{*}{$\begin{array}{l}\text { Formas participiales } \\
\text { (adjetivos y verbos) }\end{array}$} & -ádo & 107 & 50 & 106 & 50 \\
\hline & $-a ́ d a$ & 50 & 86 & 8 & 14 \\
\hline & -ído & 65 & 64 & 37 & 36 \\
\hline & $-i d a$ & 12 & 67 & 6 & 33 \\
\hline \multicolumn{6}{|c|}{$\chi^{2}=25.94>7.815 p=0,000<0,05$} \\
\hline
\end{tabular}

En formas participiales, -ádo también favorece la elisión con el 50\%, es decir, tanto en los participios como en las palabras que no lo son, el contexto -ádo es significativo para la elisión de acuerdo con los valores de $\chi 2$ y de $p$.

En un tercer análisis que Navarro propone sobre todo (tabla 7), el autor señala que cuando todo presenta función adjetival, la /d/ se elide un $45 \%$; el uso de la variante disminuye a $18 \%$ cuando la función todo es de no adjetivo.

Tabla 7. Frecuencias de retención y elisión en el cuantificador todo según su función sintáctica (Navarro 1995: 147)

\begin{tabular}{|l|l|l|l|l|l|}
\hline \multirow{2}{*}{ Variantes } & \multicolumn{2}{|c|}{ Adjetivo } & \multicolumn{2}{|c|}{ No adjetivo } & \multirow{2}{*}{ Total } \\
\cline { 2 - 5 } & $\mathrm{N}$ & $\%$ & $\mathrm{~N}$ & $\%$ & \\
\hline Retención & 85 & 54 & 134 & 82 & 219 \\
\hline Elisión & 98 & 45 & 30 & 18 & 128 \\
\hline Total & 183 & 100 & 164 & 100 & 347 \\
\hline$\chi^{2=} 46.18 p=0,000<0,05$ \\
\hline
\end{tabular}

12 El cálculo de la prueba $\chi 2$ es nuestro. 
Por último, en cuanto a los factores sociales, los resultados de Navarro (tabla 8) coinciden con los planteamientos de D' Introno y Sosa (1979) para la comunidad caraqueña: los hombres producen más elididas que las mujeres y son los hablantes de menor nivel sociocultural quienes más eliden.

Tabla 8. Las variantes de -/d/- según las variables sociales en el habla de Puerto Cabello (Basado en Navarro 1995: 137-138)

\begin{tabular}{|c|c|c|c|c|c|}
\hline \multirow{2}{*}{ Edad } & \multicolumn{2}{|c|}{ Retención } & \multicolumn{2}{|c|}{ Elisión } & \multirow{2}{*}{ Total } \\
\hline & $\mathrm{N}$ & $\%$ & $\mathrm{~N}$ & $\%$ & \\
\hline Jóvenes & 472 & 69 & 213 & 31 & 685 \\
\hline Adultos & 711 & 73 & 259 & 27 & 970 \\
\hline Total & 1183 & 71 & 472 & 29 & 1655 \\
\hline \multicolumn{6}{|c|}{$\chi^{2}=3.80<5.991 \mathrm{p}=0,051>0,05$} \\
\hline \multirow{2}{*}{ Sexo } & \multicolumn{2}{|c|}{ Retención } & \multicolumn{2}{|c|}{ Elisión } & \multirow{2}{*}{ Total } \\
\hline & $\mathrm{N}$ & $\%$ & $\mathrm{~N}$ & $\%$ & \\
\hline Hombres & 426 & 61 & 271 & 39 & 697 \\
\hline Mujeres & 757 & 79 & 200 & 21 & 957 \\
\hline Total & 1183 & 72 & 471 & 28 & 1654 \\
\hline \multicolumn{6}{|c|}{$\chi^{2}=64.02>5.991 p=0,000<0,05$} \\
\hline \multirow{2}{*}{ NSC } & \multicolumn{2}{|c|}{ Retención } & \multicolumn{2}{|c|}{ Elisión } & \multirow{2}{*}{ Total } \\
\hline & $\mathrm{N}$ & $\%$ & $\mathrm{~N}$ & $\%$ & \\
\hline I & 662 & 68 & 308 & 32 & 970 \\
\hline II & 521 & 76 & 163 & 24 & 684 \\
\hline Total & 1183 & 72 & 471 & 28 & 1654 \\
\hline \multicolumn{6}{|c|}{$\chi^{2}=12.36>5.991 p=0,000<0,05$} \\
\hline
\end{tabular}

En síntesis, en ambos estudios se confirma que el contexto fónico y los participios favorecen la elisión de -/d/- y que esta variante se proyecta como un rasgo distinguidor de los hablantes de nivel socioeconómico bajo, principalmente de los hombres. Nuestra investigación parte de estos trabajos e incorpora otros aspectos: en primer lugar, se amplían las variables lingüísticas consideradas por estos autores; la identificación de los casos, en segundo lugar, se hizo mediante el análisis espectográfico y, por último, con base en el análisis probabilístico de regresión, se determinan los factores que inciden en el debilitamiento y elisión de -/d/- en el habla caraqueña. 


\section{METODOLOGÍA}

Este estudio se inscribe en el marco del PRESEEA y sigue la Propuesta de codificación para el análisis de /d/ en posición intervocálica ${ }^{13}$ en la que se definen las variables dependientes e independientes para el estudio en cada comunidad ${ }^{14}$. Atendiendo a la Propuesta, solo se consideró la -/d/- en posición interior de palabra (cada, quedamos) y se excluyeron los casos de /d/- inicial de palabra ("la de", "aparte de") y -/d/- que siguen a una semivocal (audiovisuales [aựjoßiswáles]) o preceden a una semiconsonante (media [méơja]) ya que ambos contextos favorecen la retención. Se estudiaron 36 entrevistas del PRESEEA-Caracas (cf. Bentivoglio y Malaver 2012, 2014) de las que se extrajeron los casos de /d/ intervocálica producidos durante el comienzo (primeros cinco minutos), el intermedio (entre los minutos 25 y 30) y el final (entre el minuto 40 y el 45). Los hablantes están estratificados de acuerdo con el sexo, la edad y el grado de instrucción (tabla 9) ${ }^{15}$.

13 Proyecto coordinado "Estudio sociolingüístico del español de Granada, Las Palmas, Lleida, Madrid-Alcalá, Málaga, Sevilla y Valencia”. En Samper et al. (2010) se explica exhaustivamente la evolución de las distintas propuestas de análisis presentadas por los equipos españoles de Málaga, Granada y Las Palmas. Para tener más detalles sobre la Propuesta de Codificación véase http://www.linguas.net/LinkClick.aspx?fileticket=Z18KCe2tqLc\%3D\&t abid=474\&mid=1316 [Consulta: 21 diciembre 2014].

14 Para los resultados de los estudios peninsulares (en el marco del proyecto general PRESEEA) cf. los trabajos de Moya Corral (2012), Moya et al. (2012) y Moya Corral y García Wiedemann (2009), acerca del español de Granada; el estudio de Gómez Molina y Gómez Devís (2010) sobre el español de Valencia ; los de Samper et al. (2010) y Samper et al. (2011a) con hablantes de nivel universitario y primario de Las Palmas de Gran Canaria, respectivamente; así como Samper et al. 2011b; los trabajos de Molina Martos y Paredes García $(2014,2015)$, sobre el habla madrileña (Distrito Salamanca); también a Villena Ponsoda et al. (2011) sobre el español de Málaga; Gómez Molina, Molina Martos y Paredes García (2012) presentan un estudio contrastivo de -/d/- intervocálica en las comunidades de Madrid y Valencia.

15 En Perdomo (2015) se analizaron los minutos intermedios de la muestra caraqueña; en esta investigación aumentamos los minutos con el fin de incorporar al análisis la variable estilística. 
Tabla 9. Identificación general de los hablantes del corpus $^{16}$

\begin{tabular}{|c|c|c|c|c|c|c|}
\hline \multirow[t]{2}{*}{ GI } & \multicolumn{2}{|c|}{$\begin{array}{l}\text { Grupo etario } 1 \\
20-34 \text { años }\end{array}$} & \multicolumn{2}{|c|}{$\begin{array}{l}\text { Grupo etario } 2 \\
34-55 \text { años }\end{array}$} & \multicolumn{2}{|c|}{$\begin{array}{l}\text { Grupo etario } 3 \\
55 \text { años y más }\end{array}$} \\
\hline & Hombres & Mujeres & Hombres & Mujeres & Hombres & Mujeres \\
\hline \multirow[b]{2}{*}{1} & CARA & $\begin{array}{l}\text { CARA_ } \\
\text { M11_008 }\end{array}$ & $\begin{array}{l}\text { CARA } \\
\text { H21_015 }\end{array}$ & $\begin{array}{l}\text { CARA_ } \\
\text { M21_020 }\end{array}$ & $\begin{array}{l}\text { CARA } \\
\text { H31_028 }\end{array}$ & $\begin{array}{l}\text { CARA } \\
\text { M31_032 }\end{array}$ \\
\hline & $\begin{array}{l}\text { H11_003 } \\
\text { CARA_- } \\
\text { H11_004 }\end{array}$ & $\begin{array}{l}\text { CARA_ } \\
\text { M11_010 }\end{array}$ & $\begin{array}{l}\text { CARA_ } \\
\text { H21_016 }\end{array}$ & $\begin{array}{l}\text { CARA_ } \\
\text { M21_021 }\end{array}$ & $\begin{array}{l}\text { CARA } \\
\text { H31_029 }\end{array}$ & $\begin{array}{l}\text { CARA_ } \\
\text { M31_033 }\end{array}$ \\
\hline \multirow[b]{2}{*}{2} & CARA & $\begin{array}{l}\text { CARA } \\
\text { M12_043 }\end{array}$ & $\begin{array}{l}\text { CARA } \\
\text { H22_049 }\end{array}$ & $\begin{array}{l}\text { CARA } \\
\text { M22_055 }\end{array}$ & $\begin{array}{l}\text { CARA } \\
\text { H32_062 }\end{array}$ & $\begin{array}{l}\text { CARA } \\
\text { M32_068 }\end{array}$ \\
\hline & $\begin{array}{l}\text { CARA } \\
\text { H12_042 }\end{array}$ & $\begin{array}{l}\text { CARA- } \\
\text { M12_047 }\end{array}$ & $\begin{array}{l}\text { CARA } \\
\text { H22_051 }\end{array}$ & $\begin{array}{l}\text { CARA_ } \\
\text { M22_057 }\end{array}$ & $\begin{array}{l}\text { CARA } \\
\text { H32_063 }\end{array}$ & $\begin{array}{l}\text { CARA_ } \\
\text { M32_069 }\end{array}$ \\
\hline \multirow[b]{2}{*}{3} & CARA & $\begin{array}{l}\text { CARA } \\
\text { M13_080 }\end{array}$ & $\begin{array}{l}\text { CARA } \\
\text { H23_086 }\end{array}$ & $\begin{array}{l}\text { CARA } \\
\text { M23_093 }\end{array}$ & $\begin{array}{l}\text { CARA } \\
\text { H33_098 }\end{array}$ & $\begin{array}{l}\text { CARA } \\
\text { M33_104 }\end{array}$ \\
\hline & CARA & $\begin{array}{l}\text { CARA_- } \\
\text { M13_082 }\end{array}$ & $\begin{array}{l}\text { CARA } \\
\text { H23_08 }\end{array}$ & $\begin{array}{l}\text { CARA_- } \\
\text { M23_094 }\end{array}$ & $\begin{array}{l}\text { CARA } \\
\text { H33_101 }\end{array}$ & $\begin{array}{l}\text { CARA_ } \\
\text { M33_106 }\end{array}$ \\
\hline
\end{tabular}

\subsection{VARIABLES}

\subsubsection{Variable dependiente}

La variable dependiente, /d/ intervocálica interior de palabra, tiene tres variantes:

i) Variante elidida D-0 $[\varnothing]$

ii) Variante plena (dental) D-1 [ðָ]

iii) Variante debilitada o relajada D-2 [⿳̛口] $]$

16 En la tabla 9, cada hablante aparece representado por un código, el cual es el mismo empleado en el PRESEEA-Caracas y se interpreta de la siguiente forma:

Comunidad de habla: CARA_(Caracas)

SEXo: H (hombre); $\mathbf{M}$ (mujer)

EDAD: 1 (de 20 a 34 años); 2 (de 35 a 54 años); 3 (de 55 años en adelante).

Grado de instrucción: 1 (5 años de escolaridad); 2 (hablantes con 10-12 años de escolaridad); 3 (más de quince años de escolaridad)

IDENTIFICACIÓN DEL HABLANTE DENTRO DEL CORPUS: $\mathbf{1 0 4}$ 


\subsubsection{Variables independientes}

En cuanto a las variables independientes, se consideraron las siguientes:

i) Lingüísticas: acento; número de sílabas por palabra; estructura acentual de la palabra; entorno vocálico previo; entorno vocálico posterior; entorno consonántico en la palabra; entorno consonántico secuencial; categoría léxica; categoría gramatical (morfológica); homofonía; funcionalidad; límite morfemático; difusión léxica; asimilación al turno de palabra anterior.

ii) Estilísticas: fase de interacción

iii) Sociales: sexo; edad; nivel de estudios.

Los datos fueron analizados de acuerdo con el modelo teórico de elisión 1 en el que se contrasta la suma de todas las realizaciones de /d/ con las de su pérdida $(\partial+, \emptyset)$ : "Este es el modelo teórico que a priori se había indicado como el más adecuado para la realización de las comparaciones interdialectales" (Samper et al. 2010: 12). Para analizar las características acústicas de cada uno de los casos seleccionados se usó el programa Praat (Boersma y Weenink 2009), asimismo, se empleó el GoldVarb X (Sankoff et al. 2005) para el análisis de regresión ${ }^{17}$.

\section{RESULTADOS GENERALES}

De 540 minutos se extrajeron 3648 casos de /d/ intervocálica cuya distribución (gráfico 1) muestra que la variante elidida alcanza el 54\% de las

17 Para el análisis estadístico y probabilístico hemos hecho uso del programa GoldVarb $\mathrm{X}$, el cual facilita los valores de las frecuencias absolutas y relativas, al tiempo que, con el análisis de regresión, proporciona datos probabilísticos significativos, como la probabilidad logística escalonada, la cual permite evaluar comparativamente la importancia probabilística de cada variante sobre la variable dependiente (las variantes que tengan valores superiores 0.500 serán significativas y mientras el número se acerque más a 1, esta variante tendrá un mayor peso explicativo). El GoldVarb también ofrece la significación (cuyo valor no debe ser superior a 0.05 y cercano a 0 , con lo que indica que la relación entre las variables escogidas no es producto del azar), el input (la probabilidad media de que se produzca un fenómeno lingüístico) y el logaritmo de verosimilitud. De igual manera, se indica en los cuadros el valor del $\chi 2$ para cada uno de ellos, con lo que evaluamos si la relación entre la variable dependiente y la independiente es estadísticamente significativa. 
realizaciones, la variante debilitada, el $28 \%$ y la variante plena, el $18 \%$; el contraste de los datos del Modelo general (tres realizaciones) con los datos del Modelo elisión (dos realizaciones) confirma que el habla caraqueña se distingue por las realizaciones debilitadas $[\stackrel{\sigma}{]}]$ y elididas $[\varnothing]$ (tabla 10).

Gráfico 1. Realizaciones de la /d/ intervocálica en Caracas

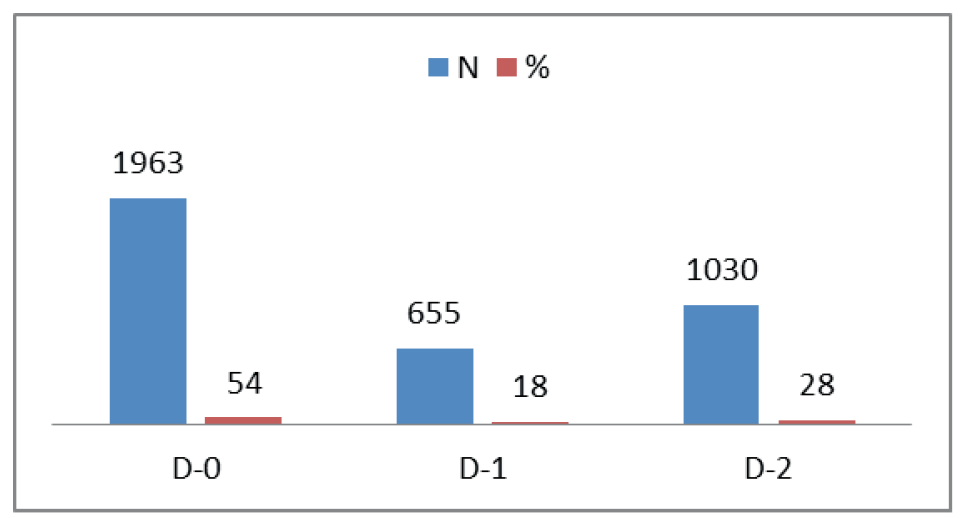

Tabla 10. Realizaciones de la/d/ intervocálica en Caracas

\begin{tabular}{|l|c|c|c|}
\cline { 2 - 3 } \multicolumn{1}{c|}{} & \multicolumn{2}{|c|}{ Modelo general } & Modelo elisión \\
\cline { 2 - 3 } \multicolumn{1}{c|}{} & $\mathrm{N}$ & $\%$ & $\%$ \\
\hline Elidida D-0 $[\varnothing]$ & 1963 & 54 & 54 \\
\hline Plena D-1 [ðণ] & 655 & 18 & \multirow{2}{*}{46} \\
\hline Debilitada D-2 $\left[{ }^{\varnothing}\right]$ & 1030 & 28 & \\
\hline Total & 3648 & 100 & 100 \\
\hline
\end{tabular}

Al reunir los resultados de las investigaciones precedentes sobre /d/ en Venezuela (gráfico 2) constatamos que la pérdida ha avanzado considerablemente, haciendo la salvedad de que los estudios de D' Introno y Sosa (1979) y Navarro (1995) se limitaron al entorno [vocal tónica]_[vocal átona]. 
Gráfico 2. Comparación temporal de la variante elidida $[\varnothing]$ en el español venezolano (\%)

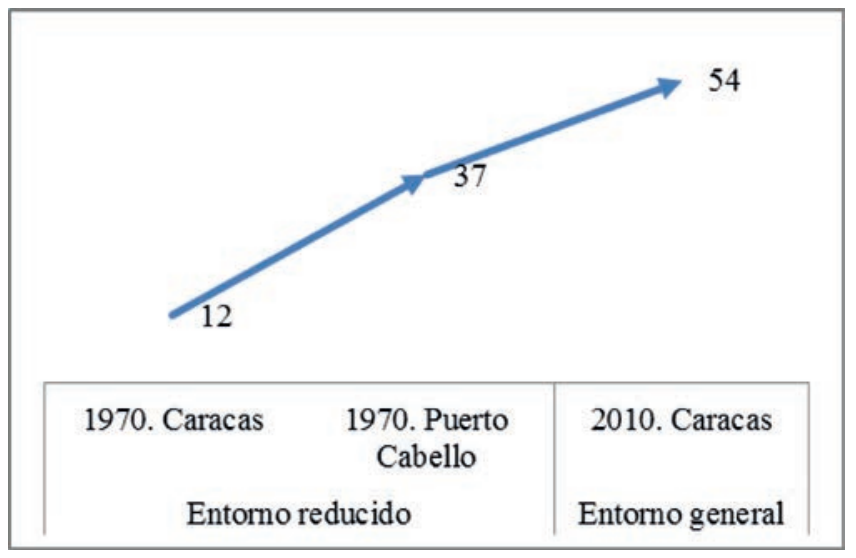

De igual modo, la comparación con el índice de elisión de las ciudades españolas muestra que Caracas está cercana a las comunidades de Granada (36\%) y Málaga (33,6\%) y alejada de Madrid (14\%), Valencia (14\%) y Las Palmas (18\%), por lo que parece representar el estado más avanzado de la alternancia retención/debilitamiento a favor de la elisión.

Con base en las frecuencias absolutas y relativas, como también en los valores probabilísticos, a continuación discutimos la incidencia de las variables lingüísticas, estilísticas y sociales en la elisión de -/d/-.

\section{RESULTADOS MODELO ELISIÓN}

\subsection{ACENTO}

Tabla 11. Resultados según la posición del acento

\begin{tabular}{|l|c|c|c|c|}
\cline { 2 - 5 } \multicolumn{1}{c|}{} & $\mathrm{N}$ & $\mathrm{D}-1$ & $\mathrm{D}-0$ & PROB \\
\hline Precede mediatamente & 2556 & 37 & 63 & $\mathbf{0 . 6 8 2}$ \\
\hline Precede inmediatamente & 36 & 47 & 53 & $\mathbf{0 . 5 8 2}$ \\
\hline Sigue mediatamente & 163 & 52 & 48 & $\mathbf{0 . 5 3 9}$ \\
\hline Sigue inmediatamente & 55 & 67 & 33 & 0.378 \\
\hline
\end{tabular}




\begin{tabular}{|l|r|r|r|r|}
\hline Sílaba tónica & 838 & 73 & 27 & 0.321 \\
\hline $\begin{array}{l}\chi^{2}=337.95>9.488 p=0,00<0,05 \\
\text { Input: } 0.445\end{array}$ \\
\hline
\end{tabular}

La posición del acento (tabla 11) incide significativamente en el proceso de elisión (0.000). Un $63 \%$ de casos de /d/ elidida se produce cuando el acento la precede inmediatamente y un $53 \%$, cuando lo hace mediatamente. La probabilidad de que ocurra la elisión en estos dos contextos es de 0.682 y de 0.582 , respectivamente. Con una probabilidad menor, de 0.539 , la elisión ocurre si el acento sigue inmediatamente a /d/; este resultado se debe, en nuestra opinión, a que las palabras todavía y además tienen frecuencias de elisión muy altas en el PRESEEA-Caracas ${ }^{18}$, pero, el valor probabilístico tan cercano a 0.500 nos dice que su incidencia en el debilitamiento de /d/ es mínima.

\subsection{NÚMERO DE SÍlABAS}

Tabla 12. Resultados según el número de sílabas

\begin{tabular}{|l|c|c|c|c|}
\cline { 2 - 5 } \multicolumn{1}{c|}{} & $\mathrm{N}$ & $\mathrm{D}-1$ & $\mathrm{D}-0$ & PROB \\
\hline Bisílaba & 1581 & 37 & 63 & $\mathbf{0 . 6 1 0}$ \\
\hline Trisílaba & 1017 & 52 & 48 & 0.458 \\
\hline Polisílaba & 1050 & 55 & 45 & 0.430 \\
\hline $\begin{array}{l}\chi^{2}=100.404>5.991 p=0,00<0,05 \\
\text { Input: } 0.523\end{array}$
\end{tabular}

Los datos recogidos en la tabla 12 confirman la importancia de esta variable en el fenómeno bajo estudio: las palabras bisílabas (queda, pude) alcanzan el $63 \%$ de elisión y es esta variante la única que tiene incidencia probabilística en la elisión (0.610). Aunque los porcentajes de elisión de las palabras trisílabas (sábados, comedor) y polisílaba (continuidad) no son bajos ( $48 \%$

18 A pesar de que en otras comunidades puede resultar pertinente recodificar esta variable, sí resulta pertinente en la comunidad caraqueña el análisis de los entornos mediatos, ya que en el contexto que precede a la consonante presentó valores significativos, tanto si el acento se halla mediata como inmediatamente. Esto, como veremos más adelante, complementa los resultados que se obtienen de la variable entorno vocálico previo. 
y $45 \%$, respectivamente), los valores probabilísticos no son significativos. En estos resultados incide con seguridad el que palabras como todo, cada, nada tuvieron altas frecuencias de aparición y elisión en todo el corpus.

\subsection{Estructura ACENTUAL DE LA PALABra}

Tabla 13. Resultados según la estructura acentual de la palabra

\begin{tabular}{|l|c|c|c|c|}
\cline { 2 - 5 } \multicolumn{1}{c|}{} & $\mathrm{N}$ & $\mathrm{D}-1$ & $\mathrm{D}-0$ & PROB \\
\hline Proparoxítona & 93 & 60 & 40 & 0.484 \\
\hline Paroxítona & 3079 & 42 & 58 & $\mathbf{0 . 6 6 5}$ \\
\hline Oxítona & 476 & 73 & 23 & 0.349 \\
\hline $\begin{array}{l}\chi^{2}=165.734>5.991 p=0,00<0,05 \\
\text { Input } 0.413\end{array}$
\end{tabular}

La estructura acentual (tabla 13) mantiene una decisiva influencia en la pérdida de -/d/-; en el PRESEEA-Caracas, la elisión tiene una probabilidad de 0.665 en palabras paroxítonas (58\%), las cuales, además, tienen $84,37 \%$ de frecuencia en el corpus (3078/3648). Por el contrario, las palabras proparoxítonas y oxítonas favorecen la retención, principalmente las oxítonas (0.349). Las palabras paroxítonas predominaron, de igual modo, en Las Palmas (20,5\%), Madrid (94,5\%) y Granada (41,4\%).

\subsection{ENTORNO VOCÁLICO PREVIO}

Tabla 14. Resultados según el entorno vocálico previo

\begin{tabular}{|c|c|c|c|c|}
\cline { 2 - 5 } \multicolumn{1}{c|}{} & $\mathrm{N}$ & $\mathrm{D}-1$ & $\mathrm{D}-0$ & PROB \\
\hline$[\mathrm{i}]$ & 323 & 74 & 26 & 0.298 \\
\hline$[\mathrm{u}]$ & 77 & 73 & 27 & 0.312 \\
\hline$[\mathrm{e}]$ & 314 & 69 & 31 & 0.351 \\
\hline$[\mathrm{o}]$ & 144 & 50 & 50 & $\mathbf{0 . 5 4 7}$ \\
\hline$[\mathrm{a}]$ & 260 & 65 & 35 & 0.390 \\
\hline$[i ́ \mathrm{i}]$ & 481 & 65 & 35 & 0.389 \\
\hline$[\mathrm{u}]$ & 48 & 58 & 42 & 0.463 \\
\hline$[\mathrm{e}]$ & 270 & 33 & 67 & $\mathbf{0 . 7 1 1}$ \\
\hline
\end{tabular}




\begin{tabular}{|c|c|c|c|c|}
\hline [ó] & 742 & 27 & 73 & $\mathbf{0 . 7 6 8}$ \\
\hline [á] & 989 & 30 & 70 & $\mathbf{0 . 7 3 4}$ \\
\hline $\begin{array}{l}\chi^{2}=534.736>16.91 p=0,00<0,05 \\
\text { Input } 0.453\end{array}$
\end{tabular}

Como se interpreta de la tabla 14, en el entorno vocálico previo predominaron las vocales tónicas un $69 \%(2530 / 3648)$ y son estas vocales las que favorecen la elisión de /d/ como muestran los pesos probabilísticos arrojados por el GoldVarb: con la vocal $o ́$ la probabilidad es de 0.768 , con $a ́$ es de 0.734 y con é la probabilidad es de 0.711 . Las vocales cerradas, por el contrario, no propician el debilitamiento, como se ve en los porcentajes de $u(42 \%)$ y de $i(35 \%)$. Entre las vocales átonas, la $o$ mostró un porcentaje de $50 \%$ de elisiones y una probabilidad de 0.547 , lo que confirma que el entorno formado odo no solo es el más frecuente sino el de mayor elisión en el corpus. Así pues, la jerarquía de las vocales previas se manifiesta como sigue: [ó] $73>$ [á] $70>$ [é] $67>$ [o] $50>$ [ú] $42>$ [i] $38>$ [í] $35>$ [a] $35>$ [e] $30>$ [u] 27 , de lo que se observa que la vocal $u$ átona es la que más favorece la retención de -/d/-.

\subsection{ENTORNO VOCÁLICO POSTERIOR}

Tabla 15. Resultados según el entorno vocálico posterior

\begin{tabular}{|c|c|c|c|c|}
\hline & $\mathrm{N}$ & $\mathrm{D}-1$ & $\mathrm{D}-0$ & PROB \\
\hline$[\mathrm{i}]$ & 81 & 52 & 48 & $\mathbf{0 . 6 0 6}$ \\
\hline$[\mathrm{u}]$ & 43 & 74 & 26 & 0.363 \\
\hline$[\mathrm{e}]$ & 277 & 41 & 59 & $\mathbf{0 . 7 0 3}$ \\
\hline$[\mathrm{o}]$ & 1500 & 35 & 65 & $\mathbf{0 . 7 5 3}$ \\
\hline$[\mathrm{a}]$ & 914 & 39 & 61 & $\mathbf{0 . 7 1 8}$ \\
\hline$[$ [í] & 99 & 72 & 28 & 0.396 \\
\hline$[$ ú] & 16 & 81 & 19 & 0.277 \\
\hline$[$ [é] & 195 & 65 & 35 & 0.471 \\
\hline [ó] & 125 & 76 & 24 & 0.344 \\
\hline [á] & 398 & 76 & 24 & 0.346 \\
\hline$\chi^{2}=352.845>16.91 p=0,00<0,05$ \\
Input 0.376
\end{tabular}


En la tabla 15 se muestra que en posición posterior al segmento dental predominan las vocales átonas en un 77\% (2815/3648). Se trata de una variable significativa (0.000) a partir de la cual se confirma que el contexto de elisión de /d/ excluye las vocales acentuadas. La $o$ es la vocal átona que más favorece la pérdida del fonema (0.753), seguida de $a$, con una probabilidad de $0.718, e$ con $0.703 \mathrm{y}$, por último, $i$ con 0.606 .

\subsection{ENTORNO CONSONÁNTICO EN LA PALABRA ${ }^{19}$}

Tabla 17. Resultados según el entorno consonántico en la palabra

\begin{tabular}{|l|c|c|c|c|}
\cline { 2 - 5 } \multicolumn{1}{c|}{} & $\mathrm{N}$ & $\mathrm{D}-1$ & $\mathrm{D}-0$ & PROB \\
\hline No hay en el entorno $/ \mathrm{d} /$ & 3507 & 46 & 54 & $\mathbf{0 . 6 3 7}$ \\
\hline$/ \mathrm{d} /$ previa realizada como $\left[{ }^{\ominus}\right]$ & 56 & 48 & 52 & $\mathbf{0 . 6 1 2}$ \\
\hline$/ \mathrm{d} /$ previa elidida & 15 & 33 & 67 & $\mathbf{0 . 7 4 6}$ \\
\hline$/ \mathrm{d} /$ posterior realizada como $\left[{ }^{\circ}\right]$ & 33 & 85 & 15 & 0.208 \\
\hline$/ \mathrm{d} /$ posterior elidida & 37 & 76 & 24 & 0.320 \\
\hline $\begin{array}{l}\chi^{2}=34.475>9.488 p=0,00<0,05 \\
\text { Input } 0.406\end{array}$
\end{tabular}

La primera variante de esta variable refiere a la ausencia de otro segmento dental en la palabra y en esos casos (tabla 17), la elisión posee una probabilidad de 0.637 . La presencia previa de una /d/ elidida dentro de la palabra aumenta el índice a 0.746 , un valor predictivo bastante alto. Las variantes $/ \mathrm{d} /$ posterior elidida y $/ \mathrm{d} /$ posterior realizada como $\left[{ }^{\natural}\right]$ son las que presentan los índices más bajos de pérdida de la consonante, con un $24 \%$ y un $15 \%$, respectivamente. Estos resultados indican que en aquellas palabras en las que hay más de un caso de /d/ intervocálica, la segunda en realizarse tenderá a elidirse, en especial si es precedida por otra elidida. 


\subsection{ENTORNO CONSONÁNTICO SECUENCIAL}

Tabla 18. Resultados según el entorno consonántico secuencial

\begin{tabular}{|l|l|l|l|l|}
\cline { 2 - 6 } \multicolumn{1}{c|}{} & $\mathrm{N}$ & $\mathrm{D}-1$ & $\mathrm{D}-0$ & PROB \\
\hline No hay en el entorno /d/ & 1891 & 48 & 52 & 0.480 \\
\hline $\begin{array}{l}\text { Hay en el entorno consonántico secuencial } \\
\text { una o más /d/ realizada(s) como [d] }\end{array}$ & 743 & 49 & 51 & 0.467 \\
\hline $\begin{array}{l}\text { Hay en el entorno consonántico secuencial } \\
\text { una o más /d/ elididas }\end{array}$ & 1014 & 41 & 59 & $\mathbf{0 . 5 5 3}$ \\
\hline $\begin{array}{l}\chi^{2}=17.818>5.991 p=0,00<0,05 \\
\text { Input } 0.541\end{array}$ & & \multicolumn{4}{|l}{} \\
\hline
\end{tabular}

Tal como se presenta en la tabla 18, se clasificaron los casos en función de la presencia o ausencia de realizaciones elididas en el grupo fónico (secuencia de sonidos entre dos pausas). La elisión se da en todos los contextos considerados, particularmente si, como se indica en la tabla 18 , en el entorno secuencial hay otros casos de elisión (0.553).

Los resultados de las variables entorno consonántico en la palabra y entorno consonántico secuencial muestran que en la realización de la variante $[\varnothing]$ incide un proceso de asimilación consonántica, ya que cuando en el entorno hay otros casos de elididas, la variante $[\varnothing]$ tiene mayores probabilidades de producirse.

Hasta aquí, como se ha discutido, las variables fonéticas propuestas para el estudio del debilitamiento de -/d/-. En lo que sigue se discuten los resultados de las variables morfológicas, léxicas y sociales.

\subsection{CATEGORÍA LÉXICA}

Tabla 19. Resultados según la categoría léxica

\begin{tabular}{|l|c|c|}
\cline { 2 - 3 } \multicolumn{1}{c|}{} & $\mathrm{N} / \mathrm{T}$ & $\%$ \\
\hline Nombre propio & $47 / 107$ & 44 \\
\hline Término & $38 / 95$ & 40 \\
\hline Otros & $1878 / 3446$ & 54 \\
\hline$\chi^{2}=5.037>5.991 p=0,00<0,05$ \\
\hline
\end{tabular}


En Caracas esta variable no resultó significativa, dados los valores de $\chi^{2}$, que se aprecian en la tabla 19. La variante que más casos tiene es otros con $54 \%$ de casos elididos (todo, madera, maldades). Los nombres propios (Madariaga) favorecen la realización plena de la /d/ en el 66\% de los casos (107) y de los 95 casos clasificados como términos, el $40 \%$ se producen con la variante elidida.

\subsection{CATEgoría gRAMATical (MORFológica)}

Tabla 20. Resultados según la categoría gramatical

\begin{tabular}{|l|c|c|c|c|}
\cline { 2 - 5 } \multicolumn{1}{c|}{} & $\mathrm{N}$ & $\mathrm{D}-1$ & $\mathrm{D}-0$ & PROB \\
\hline Verbo-participio & 364 & 44 & 56 & 0.462 \\
\hline Otras formas del verbo & 585 & 49 & 51 & 0.415 \\
\hline Nombre & 1077 & 64 & 36 & 0.276 \\
\hline Adjetivo & 506 & 48 & 52 & 0.423 \\
\hline Pronombre & 552 & 30 & 70 & $\mathbf{0 . 6 1 0}$ \\
\hline Adverbio & 120 & 38 & 63 & $\mathbf{0 . 5 2 8}$ \\
\hline Determinante & 358 & 19 & 81 & $\mathbf{0 . 7 3 8}$ \\
\hline Otros & 86 & 36 & 64 & $\mathbf{0 . 5 4 4}$ \\
\hline$\chi^{2}=306.23>14.067 p=0,00<0,05$ \\
\hline
\end{tabular}

En el PRESEEA-Caracas, determinantes y pronombres lideran el proceso de elisión. En la tabla 20 se aprecia que el determinante favorece la elisión con un índice de probabilidad de 0.738 y el pronombre con 0.610 . El adverbio, en tercer lugar, muestra 0.528 de probabilidad de ser elidido. En la categoría Otros (modo, lado, navidad) se observa $64 \%$ de elisiones y una probabilidad de 0.544 que da cuenta de que hay casos de elisión que no son azarosos.

Entre las categorías que no favorecen la elisión en el habla caraqueña, se encuentra, en primer lugar, el nombre con el $64 \%$ de plenas $(0.276)$, en segundo lugar, el adjetivo, con $52 \%$ de elisiones y un índice de probabilidad de $0.423 \mathrm{y}$, en tercer lugar, el participio, que tampoco favorece la variante $[\emptyset]$ en el PRESEEA-Caracas (0.462). Al comparar los datos caraqueños con los de las otras comunidades del PRESEEA encontramos que también en Las Palmas el participio se ubicó por debajo del determinante, con el $26 \%$, y en la comunidad valenciana obtuvo un $26 \%$ de elisiones (dos puntos por debajo del determinante); por el contrario, en el Distrito de Salamanca, Madrid, el 50,77\% de elisiones son participios, en Granada, el 62,6\% y en 
Málaga, el 66\%. Vistos estos resultados, las comunidades pueden organizarse en dos grupos: a) comunidades en las que la elisión se da en el participio (Granada, Málaga y Madrid) y b) comunidades en las que la elisión se da en el determinante (Caracas, Canarias, Valencia).

\subsection{Homofonía (EXISTENCIA DE HOMÓFONO DE DIFERENTE CATEGORÍA MORFOLÓGICA O FUNCIONAL)}

Tabla 21. Resultados según la homofonía

\begin{tabular}{|l|l|l|l|l|}
\cline { 2 - 6 } \multicolumn{1}{c|}{} & $\mathrm{N}$ & $\mathrm{D}-1$ & $\mathrm{D}-0$ & PROB \\
\hline Ausencia de homofonía & 1841 & 56 & 44 & 0.441 \\
\hline Homofonía entre nombre o adjetivo y participio & 464 & 40 & 60 & $\mathbf{0 . 5 9 8}$ \\
\hline Homofonía entre adjetivo y nombre & 275 & 59 & 41 & 0.411 \\
\hline Homofonía entre sustantivo y verbo & 66 & 68 & 32 & 0.315 \\
\hline Otros casos de homofonía & 1002 & 27 & 73 & $\mathbf{0 . 7 2 6}$ \\
\hline $\begin{array}{l}\chi^{2}=250.07>9.488 p=0,00<0,05 \\
\text { Input } 0.504\end{array}$ & \multicolumn{5}{|c}{} \\
\hline
\end{tabular}

La finalidad de examinar esta variable es constatar si la homofonía puede contribuir a la elisión de la /d/ y, efectivamente, ocurre que la pérdida del segmento dental está en relación con esta variable (0.000). La Homofonía entre nombre o adjetivo y participio (pasado) arroja un moderado índice de 0.598 mientras que Otros casos de homofonía (todo, nada) alcanza un peso notorio y se debe a que esta categoría agrupa palabras bisílabas como todo, con frecuencias altas en el corpus y porcentajes elevados de elisión cuando cumple la función de determinante.

\subsection{FunCIONALIDAD}

Tabla 22. Resultados según la funcionalidad

\begin{tabular}{|l|c|c|c|c|}
\cline { 2 - 5 } \multicolumn{1}{c|}{} & $\mathrm{N}$ & $\mathrm{D}-1$ & $\mathrm{D}-0$ & PROB \\
\hline No existe funcionalidad & 3130 & 46 & 54 & $\mathbf{0 . 5 9 4}$ \\
\hline $\begin{array}{l}\text { La/d/marca virtualmente diferencia } \\
\text { semántica en un par mínimo de } \\
\text { diferente categoría morfológica }\end{array}$ & 384 & 35 & 65 & $\mathbf{0 . 6 9 5}$ \\
\hline
\end{tabular}




\begin{tabular}{|l|l|l|l|l|}
\hline $\begin{array}{l}\text { La/d/marca virtualmente diferencia } \\
\text { semántica en un par mínimo de la } \\
\text { misma categoría morfológica }\end{array}$ & 134 & 81 & 19 & 0.231 \\
\hline $\begin{array}{l}\chi^{2}=81.78>5.991 p=0,00<0,05 \\
\text { Input } 0.445\end{array}$ \\
\hline
\end{tabular}

Cuando la /d/ hace la distinción entre un par mínimo de la misma categoría gramatical, la elisión se frena como señala el $19 \%$ de $[\varnothing]$ en pares como boa/ boDa, moho/moDo. Ahora bien, en la tabla 22 también se aprecia que [ $\varnothing]$ se produce independientemente de si la variación retención/elisión afecta la distinción semántica del par mínimo (0.695). Cabe esperar que la frecuencia de todo influya en estos resultados, ya que de los 384 casos de la variante, 194 corresponden a todos, y 149 de ellos son elididos. La variante No existe funcionalidad muestra un valor probabilístico de 0.594 , lo que significa que su incidencia no es muy alta.

\subsection{LÍMITE MORFEMÁTICO}

Tabla 23. Resultados según el límite morfemático

\begin{tabular}{|l|c|c|}
\cline { 2 - 3 } \multicolumn{1}{c|}{} & $\mathrm{N} / \mathrm{T}$ & $\%$ \\
\hline $\mathrm{La} / \mathrm{d} /$ en el lexema & $1306 / 2395$ & 55 \\
\hline $\mathrm{La} / \mathrm{d} /$ en el morfema o participio & $657 / 1253$ & 52 \\
\hline$\chi 2=1.454>3.841 \mathrm{p}=0.22<0,05$ \\
\hline
\end{tabular}

Esta variable no aporta ninguna explicación a la elisión ya que en ambos casos los porcentajes son casi idénticos y el $\chi^{2}$ no es significativo.

\subsection{DiFUSIÓN LÉXICA}

Tabla 24. Resultados según la difusión léxica

\begin{tabular}{|l|c|c|c|c|}
\cline { 2 - 5 } \multicolumn{1}{c|}{} & $\mathrm{N}$ & $\mathrm{D}-1$ & $\mathrm{D}-0$ & PROB \\
\hline Todavía & 40 & 15 & 85 & 0.784 \\
\hline Todo (modificador) & 404 & 20 & 80 & 0.715 \\
\hline Nada & 174 & 26 & 74 & 0.640 \\
\hline
\end{tabular}




\begin{tabular}{|l|c|c|c|c|}
\hline Todo (núcleo) & 325 & 31 & 69 & 0.587 \\
\hline Poder & 236 & 35 & 65 & 0.546 \\
\hline Cada & 58 & 36 & 64 & 0.530 \\
\hline & & & & \\
\hline Además & 10 & 40 & 60 & 0.490 \\
\hline Quedar & 157 & 50 & 50 & 0.387 \\
\hline Otras & 2133 & 54 & 46 & 0.346 \\
\hline Vida & 111 & 86 & 14 & 0.091 \\
\hline $\begin{array}{l}\chi^{2}=333.33>16.919 p=0,00<0,05 \\
\text { Input } 0.610\end{array}$
\end{tabular}

Como se aprecia en la tabla 24, hay tres palabras en las que recae la elisión de forma casi categórica. Todavía arroja un peso probabilístico de 0.784 de elisión, seguida de todo (la palabra con mayor frecuencia en el corpus) que cuando cumple la función de modificador manifiesta un índice probabilístico de 0.715. Nada, la tercera variante en el PRESEEA-Caracas, alcanzó un peso de 0.640 . Por otra parte, y aunque con pesos probabilísticos muy cercanos a 0.500 , destacan todo (función núcleo), el verbo poder y cada.

Además y quedar favorecen la retención, aunque el adverbio un poco más. Vida es un contexto en el que la retención es categórica, lo que debe tomarse en cuenta para análisis futuros de esta variable en concordancia con lo que se comentó acerca de la categoría gramatical (tabla 21) y sobre el entorno vocálico ida ( $27 \%$ de elisión). Nuestros resultados se asemejan a los de Las Palmas (Samper et al. 2011b) donde la variante elidida obtuvo en todo (modificador) una probabilidad de 0.838 , como también en el adverbio todavía, con una probabilidad de 0.731 .

\subsection{Asimilación al tURNo De PALABRA ANTERIOR}

Tabla 25. Resultados según la asimilación al turno de palabra anterior

\begin{tabular}{|l|c|c|}
\cline { 2 - 3 } \multicolumn{1}{l|}{} & N/T & $\%$ \\
\hline $\begin{array}{l}\text { No se produjo ninguna aparición cercana } \\
\text { de /d/ en el turno anterior }\end{array}$ & $1641 / 3049$ & 54 \\
\hline $\begin{array}{l}\text { En el turno de palabra anterior se } \\
\text { produjeron una o más ocurrencias de /d/ } \\
\text { elidida }\end{array}$ & $173 / 302$ & 57 \\
\hline
\end{tabular}




\begin{tabular}{|l|c|c|}
\hline $\begin{array}{l}\text { En el turno de palabra anterior se } \\
\text { produjeron una o más ocurrencias de /d/ }\end{array}$ & $149 / 297$ & 50 \\
\hline$\chi^{2}=3.052>5.991 p=0,00<0,05$ \\
\hline
\end{tabular}

Si bien el valor de $\chi^{2}$ en la tabla 25 indica que se trata de una variable poco significativa, no deja de ser útil comentar las frecuencias de cada variante. Un 57\% de elisiones ocurre cuando el interlocutor elide y un 54\% cuando no hace uso de -/d/-. Un 50\% de elisiones cuando en el turno de palabra anterior hay casos de /d/ retenida. Estos resultados evidencian que el hablante elide independientemente de la pronunciación del interlocutor.

\subsection{VARIABLE ESTILÍSTICA: FASE DE INTERACCIÓN}

Tabla 26. Resultados según la fase de interacción

\begin{tabular}{|l|c|c|c|c|}
\cline { 2 - 5 } \multicolumn{1}{c|}{} & $\mathrm{N}$ & $\mathrm{D}-1$ & $\mathrm{D}-0$ & PROB \\
\hline Al inicio & 1180 & 49 & 51 & 0.470 \\
\hline Intermedios & 1214 & 48 & 52 & 0.486 \\
\hline Finales & 1254 & 42 & 58 & $\mathbf{0 . 5 4 4}$ \\
\hline$\chi^{2}=14.94>3.841 \mathrm{y}$ de $p=0,00<0,05$ \\
\hline
\end{tabular}

Esta variable pretende reflejar el grado de control sobre el habla que tienen los entrevistados. Se parte de la presunción de que los primeros cinco minutos de la entrevista corresponden a un registro controlado, poco espontáneo que, hacia el final de la entrevista, ha cambiado y se ha hecho más espontáneo y relajado. Los resultados en la tabla 26 muestran un aumento progresivo de la elisión de un 51\% (inicio), pasando a 52\% (intermedio de la entrevista) hasta el final, en el que se presenta el porcentaje de $58 \%$ de elididas. Es una variable de poco poder explicativo dado el peso probabilístico de 0.544 que arroja el GoldVarb, lo que confirma su poca influencia en la elisión. 
Gráfico 3. Elisión según la fase de interacción (\%)

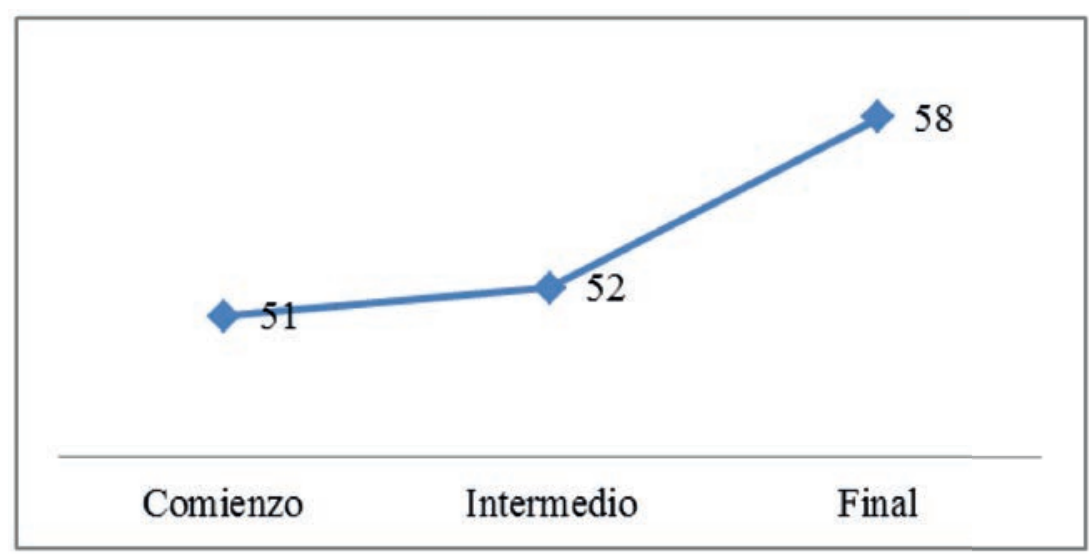

\subsection{SEXo}

Tabla 27. Resultados generales según el sexo

\begin{tabular}{|l|c|c|c|c|}
\cline { 2 - 5 } \multicolumn{1}{c|}{} & $\mathrm{N}$ & $\mathrm{D}-1$ & $\mathrm{D}-0$ & PROB \\
\hline Hombres & 1773 & 42 & 58 & $\mathbf{0 . 5 4 0}$ \\
\hline Mujeres & 1875 & 50 & 50 & 0.460 \\
\hline$\chi^{2}=24.13>3.841$ & $=$ & $0,00<0,05$ \\
\hline
\end{tabular}

En la tabla 27 se aprecia que la elisión es de un $58 \%$ entre los hombres y un $50 \%$ entre las mujeres, datos que confirman que son los hombres quienes protagonizan el proceso de cambio de la -/d/- en la comunidad caraqueña, mientras que las mujeres, con ocho puntos porcentuales menos, se hallan más cerca de lo que dicta el modelo estándar de pronunciación. La explicación de este comportamiento ha descansando en la noción del prestigio encubierto que puede representar la realización $[\varnothing]$ entre los hombres de los estratos socioeconómicos bajos, de acuerdo con Moreno Fernández (2004). Caracas coincide con las comunidades de Las Palmas, Málaga y Madrid, pues los resultados mostraron que los hombres eliden más que las mujeres. 


\subsection{EDAD}

Tabla 28. Resultados según la edad

\begin{tabular}{|l|c|c|c|c|}
\cline { 2 - 5 } \multicolumn{1}{c|}{} & $\mathrm{N}$ & $\mathrm{D}-1$ & $\mathrm{D}-0$ & PROB \\
\hline Primera generación (20-34 años) & 1195 & 38 & 62 & $\mathbf{0 . 5 8 1}$ \\
\hline Segunda generación (35-54 años) & 1275 & 47 & 53 & 0.495 \\
\hline $\begin{array}{l}\text { Tercera generación (de 55 años } \\
\text { en adelante) }\end{array}$ & 1178 & 54 & 46 & 0.424 \\
\hline $\begin{array}{l}\chi^{2}=24.13>5.991 p=0,00<0,05 \\
\text { Input } 0.539\end{array}$ & & & \\
\hline
\end{tabular}

La elisión de -/d/-, según se observa en los resultados de la tabla 28, es mayor entre los hablantes de la primera generación quienes alcanzan el $62 \%$ de los casos; la segunda generación elide en un $53 \%$ de los casos y los mayores lo hacen un $46 \%$. Así, los dos grupos intermedios manifiestan notorios porcentajes de elididas y son los de mayor edad los que más mantienen la variante dental (54\%); a pesar del comportamiento de los jóvenes, quienes alcanzan un índice probabilístico de 0.581 , debe descartarse que la elisión sea un rasgo generacional en la sincronía caraqueña; por el contrario, la frecuencia de elididas de este grupo y el hecho de que los de más edad elidan en un $46 \%$ comprueban que se trata de un comportamiento difundido. Creímos necesario conocer, además, cómo se relacionan los factores sociales en la elisión por lo que se cruzaron la edad y el sexo (tabla 29, gráfico 4) y se encontró que en los tres grupos generacionales, son los hombres los que producen la variante $[\varnothing]$; no obstante, y a pesar de que el $\chi^{2}$ no es significativo, las diferencias etarias son dignas de destacar ya que son los hombres jóvenes los que lideran el avance de la elisión.

Tabla 29. Elisión según la edad y sexo

\begin{tabular}{|l|c|c|c|c|}
\hline & Hombres & $\%$ & Mujeres & $\%$ \\
\hline 20-34 años & $394 / 565$ & 70 & $345 / 630$ & 55 \\
\hline 35-54 años & $354 / 627$ & 56 & $326 / 648$ & 50 \\
\hline 55 años en adelante & $280 / 581$ & 48 & $264 / 597$ & 44 \\
\hline & $\chi^{2}=0.46>5.991 p=0,00<0,792$ \\
\hline
\end{tabular}


Gráfico 4. Elisión según la edad y sexo (\%)

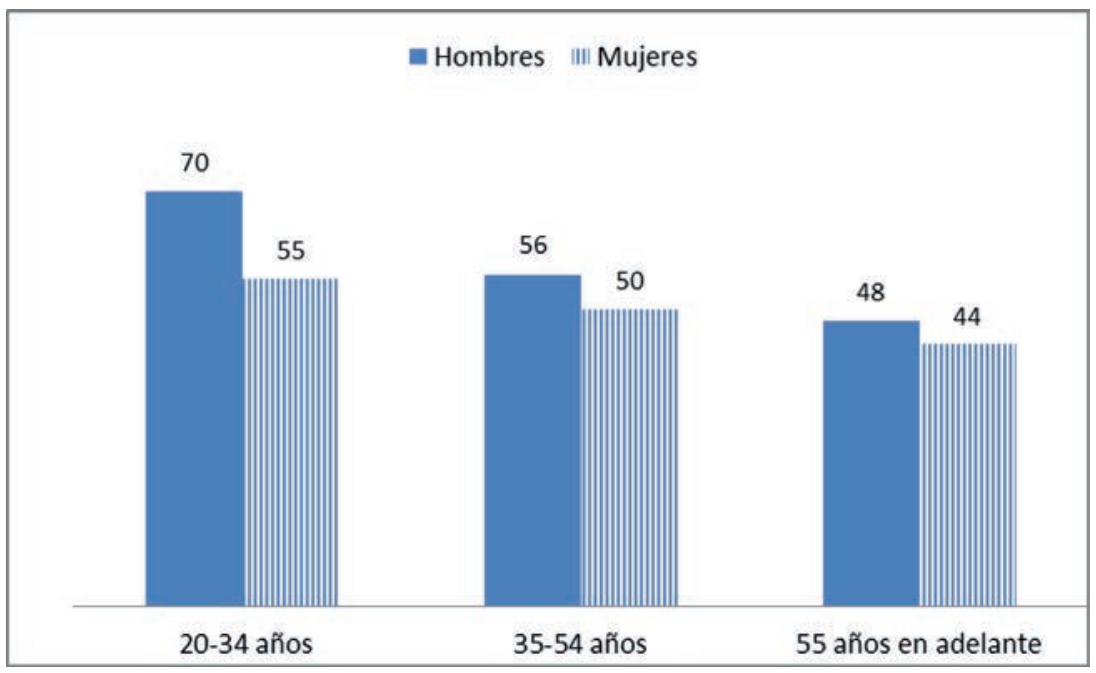

Como se puede apreciar (gráfico 4), las diferencias entre hombres y mujeres son mayores entre los jóvenes: los hombres jóvenes eliden $70 \%$ y las mujeres $55 \%$, una diferencia de 25 puntos. A medida que aumenta la edad, las diferencias disminuyen a 4 puntos entre los hablantes mayores.

\subsection{GRADO DE INSTRUCCIÓN}

Tabla 30. Resultados según el grado de instrucción

\begin{tabular}{|l|c|c|c|c|}
\cline { 2 - 5 } \multicolumn{1}{c|}{} & $\mathrm{N}$ & $\mathrm{D}-1$ & $\mathrm{D}-0$ & PROB \\
\hline Primarios & 1178 & 41 & 59 & $\mathbf{0 . 5 4 7}$ \\
\hline Secundarios & 1150 & 47 & 53 & 0.487 \\
\hline Universitarios & 1320 & 49 & 51 & 0.466 \\
\hline$\chi^{2}=17.043>5.991$ & $p=0,00<0,05$ \\
\hline
\end{tabular}

Tal como se indica en la tabla 30, los hablantes de estudios primarios son los que más eliden, con el $59 \%$ de los casos, los hablantes de estudios intermedios (secundaria completa) lo hacen en el 53\% y los hablantes de estudios universitarios lo hacen un 51\%. Si bien la elisión es un rasgo que caracteriza a los sociolectos bajos de la comunidad caraqueña, el nivel educativo no es 
una variable de gran incidencia -dado el peso de 0.547 identificado por el GoldVarb- pues se trata de un proceso debido, principalmente, a factores internos. Se relacionaron el grado de instrucción y el sexo (tabla 31, gráfico 5), y se constató que, para ambos sexos, los porcentajes más altos de elisión corresponden al grado 1 de instrucción y que los hombres con educación intermedia muestran frecuencias similares a las de las mujeres del grado 1 y grado 2 . Entre las mujeres, las más conservadoras son las mujeres con estudios universitarios.

Tabla 31. Resultados según grado de instrucción y sexo

\begin{tabular}{|l|c|c|c|c|}
\cline { 2 - 5 } \multicolumn{1}{c|}{} & Hombres & $\%$ & Mujeres & $\%$ \\
\hline Primarios & $377 / 588$ & 64 & $313 / 590$ & 53 \\
\hline Secundarios & $280 / 517$ & 54 & $326 / 633$ & 52 \\
\hline Universitarios & $371 / 668$ & 56 & $296 / 652$ & 45 \\
\hline$\chi^{2}=13.29>5.991 p=0,00<0,001$ \\
\hline
\end{tabular}

Gráfico 5. Elisión según grado de instrucción y sexo (\%)

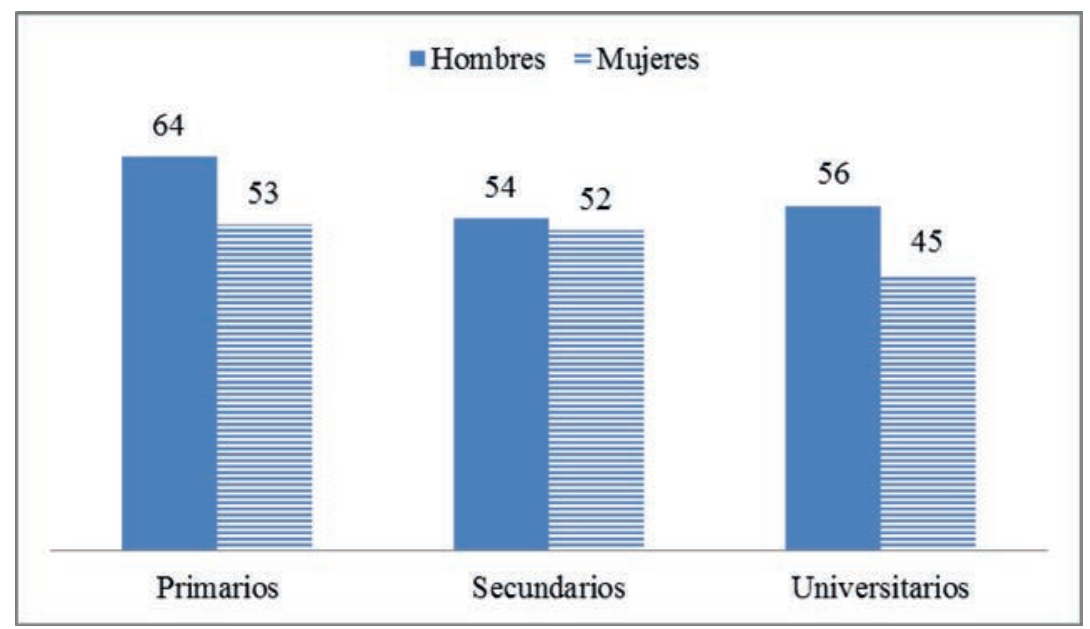

Para finalizar esta parte del análisis, se han graficado los porcentajes de elisión según el grado de instrucción y edad (gráfico 6) y según el grado de instrucción y estilo de habla (gráfico 7). 
Gráfico 6. Elisión según grado de instrucción y edad (\%)

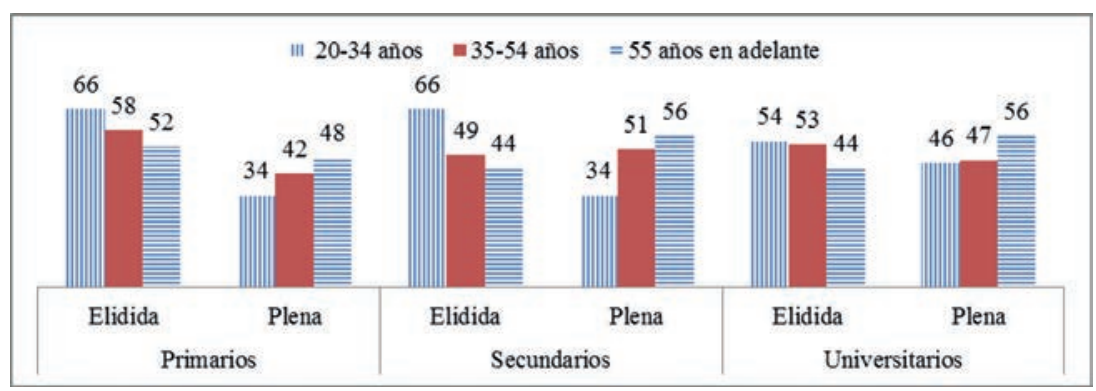

Grafico 7. Elisión según grado de instrucción y estilo (\%)

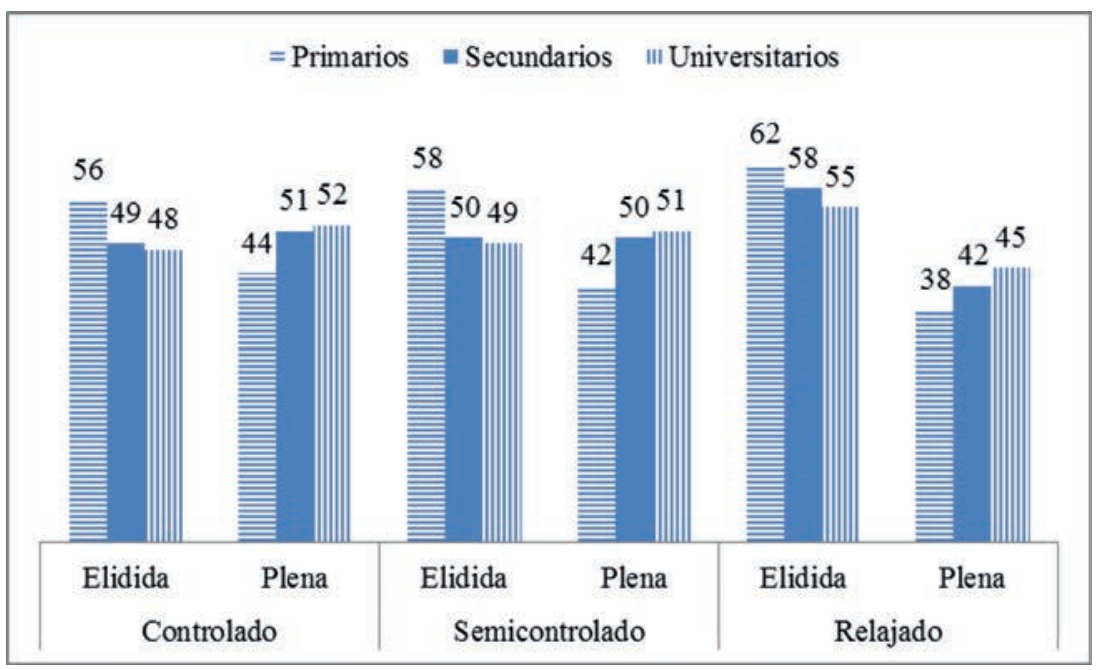

De acuerdo con ambos gráficos, $[\varnothing]$ constituye una variable sociolingüística estable (cf. Labov 1994; Silva-Corvalán 2001) por las siguientes razones: i) se distribuye en la comunidad atendiendo al criterio educativo/socioeconómico: aumenta entre los hablantes de menos estudios y nivel socioeconómico y disminuye entre los de nivel alto y más estudios; ii) covaría con el estilo: aumenta en el estilo relajado y disminuye en el estilo controlado; iii) las mujeres de mayor grado de instrucción se manifiestan como conservadoras ante el cambio y, por el contrario, los hombres de menor instrucción se muestran más innovadores. 


\section{CONCLUSIONES}

Analizados los datos de la retención y elisión de /d/ intervocálica en el entorno [vocal]__vocal] se ha comprobado que el proceso de debilitamiento muestra un notable aumento en la comunidad caraqueña.

En cuanto a los condicionantes fonéticos, la elisión ocurre cuando -/d/- aparece en palabras bisílabas, paroxítonas y el acento le precede mediatamente. La variante $[\varnothing]$ se limita principalmente a la secuencia [vocal tónica]_[vocal átona], el llamado entorno restringido, formada por las combinaciones -odo, -ado, -ede con frecuencias significativamente altas de elisión. En el plano morfológico, determinantes y pronombres encabezan el proceso de elisión en Caracas; hay que destacar que el participio no constituye el entorno más favorable para la pérdida de /d/ y, en su lugar aparece el adverbio, principalmente todavía, el cual posee una alta frecuencia de elisión en el corpus. Vistos estos resultados, la elisión parece producirse en el orden categorial siguiente:

\section{DETERMINANTES/PRONOMBRES todo $\rightarrow$ nada $\rightarrow$ ADVERBIOS todavía $\rightarrow$ PARTICIPIOS (raspao/parao/cansao/animaolalimentaolaprendio/ acostumbrao).}

Las variables sociales muestran que /d/ se elide es propia de los hombres, frecuente entre los de menor edad de la comunidad y los de estudios primarios. El análisis del tiempo aparente (Labov 1994) indica que la elisión continuará evolucionando en el seno de la comunidad de acuerdo con el patrón de estratificación social débil que manifiesta en la actualidad. La elisión se muestra muy relacionada con la frecuencia de determinadas palabras, por lo que el patrón de difusión léxica (cf. Chambers and Trudgill 1994; Labov 1994; Penny 2000; Martín Butragueño 2014) parece responder al proceso de cambio en la comunidad caraqueña.

Tabla 32. Variables significativas según el modelo probabilístico

\begin{tabular}{|l|c|c|}
\hline Variable & Log-ver & Sign. \\
\hline Entorno vocálico previo & -2243.759 & 0.000 \\
\hline Entorno vocálico posterior & -2335.968 & 0.000 \\
\hline Difusión léxica & -2341.337 & 0.000 \\
\hline Acento & -2345.702 & 0.000 \\
\hline Categoría gramatical & -2358.845 & 0.000 \\
\hline
\end{tabular}




\begin{tabular}{|l|r|l|}
\hline Número de sílabas de la palabra & -2467.435 & 0.000 \\
\hline Edad & -2488.494 & 0.000 \\
\hline Sexo & -2505.913 & 0.000 \\
\hline Nivel de estudios & -2509.450 & 0.000 \\
\hline Fase & -2510.505 & 0.001 \\
\hline
\end{tabular}

\section{REFERENCIAS BIBLIOGRÁFICAS}

Alba, Orlando. 1999. Elisión de la /d/ intervocálica postónica en el español dominicano. En Amparo Morales et al. (eds.). Homenaje a María Vaquero, 3-21. San Juan de Puerto Rico: Editorial de la Universidad de Puerto Rico.

2000. Elisión de la /d/ intervocálica postónica. En Orlando Alba, Nuevos aspectos del español en Santo Domingo, 51-72. Santo Domingo: Librería La Trinitaria/ Brigham Young University.

Bentivoglio, Paola e Irania Malaver. 2012. Corpus sociolingüístico de Caracas: PRESEEA Caracas 2004-2010. Hablantes de instrucción superior. Boletín de Lingüística 37-38: 144-180.

2014. Corpus sociolingüístico de Caracas: PRESEEA-Caracas 20042010. [CD-ROM] Caracas: Universidad Central de Venezuela

Boersma, Paul y David Weenink. 2009. Praat Doing phonetics by computer (Versión 5.4.04) [Programa para computadora].

Cano Aguilar, Rafael. 1992. El español a través de los tiempos. Madrid: Arco/Libros.

Cedergren, Henrieta. 1973. La elisión de la /d/: un ensayo de comparación dialectal. Boletín de la Academia Puertorriqueña de la Lengua Española 12-29.

Chambers, J.K y Peter Trudgill. 1994. La dialectología. Madrid: Visor Libros.

Díaz-Campos, Manuel y Michael Groville. 2011. An Analysis of Frequency as Factor Contributing to the Diffusion of Variable Phenomena: Eviden from Spanich Data. En Luis Ortiz-López (ed.). Selected Proceedings of the 13th Hispanic Linguistics Symposium, 224-238. Somerville, MA: Cascadilla.

Díaz-Campos, Manuel, Stephen Fafulas y Michael Gradoville. 2011. Going Retro: An Analysis of the Interplay between Socioeconomic Class and Age in Caracas Spanish. En Jim Michnowicz y Robin Dodsworth (eds.). Selected Proceedings of the 5th Wokshop on Spanish Sociolinguistics, 65-78. Somerville, MA: Cascadilla Proceedings Project.

D’Introno, Francesco y Juan Manuel Sosa. 1979. Elisión de la /d/ en el español de Caracas: Aspectos sociolingüísticos e implicaciones teóricas. Anuario de la Escuela de Letras UCV. 33-61.

1986. Elisión de la /d/ en el español de Caracas: Aspectos sociolingüísticos e implicaciones teóricas. En Rafael Núñez Cedeño, Iraset Páez Urdaneta y Jorge Guitart (comp.). Estudios sobre la fonología del español del Caribe, 135-165. Caracas: La Casa de Bello.

Gómez Molina, José Ramón y María Begoña Gómez Devís. 2010. Mantenimiento y elisión de la /d/ intervocálica en el español de Valencia. Verba 37: 89-122. 
Gómez Molina, José Ramón, Isabel Molina Martos y Florentino Paredes García. 2012. La variación de -/d/- intervocálica en comunidades de habla conservadoras de España: los casos de Madrid y Valencia. https://www.academia.edu/10741755/La_variaci\%C3\%B3n de_-_d_-_intervoc\%C3\%A1lica_en_comunidades_de_habla_conservadoras_de_ Espa $\% \overline{\mathrm{C}} 3 \% \mathrm{~B} 1 \mathrm{~B}$ _los_casos_de_Madrid_y_Valencia_2012_-

Labov, William. 1994. Principios del cambio lingüístico. Volumen I: Factores internos. Madrid: Gredos.

López Morales, Humberto. 1983. Estratificación social del español de San Juan de Puerto Rico. México: Universidad Nacional Autónoma de México.

Martín Butraguño, Pedro. 2014. Fonología variable del español de México, vol. 1: Procesos segmentales. México: El Colegio de México.

Márquez, Julio; Darcy Rojas y Thania Villamizar. 2006. Variantes del fonema /d/ en dos estilos de habla en Apartaderos (Mérida, Venezuela). Lingua Americana 10 ( $\left.\mathrm{N}^{\circ} 19\right)$ : 67-73.

Molina Martos, Isabel y Florentino Paredes Garcia. 2014. Sociolingüística de la elisión de la dental/d/ en Madrid (Distrito Salamanca). Cuadernos de Lingüistica 2: 55-114.

2015. La conservación de la dental -/d/- en el distrito de Salamanca. En Ana

María Cestero Mancera, Isabel Molina Martos, Florentino Paredes García (coord.). Patrones sociolingüísticos de Madrid, 63-89, Suiza: Peter Lang.

Moreno Fernández, Francisco. 2004. Cambios vivos en el plano fónico del español: variación dialectal y sociolingüística. En Rafael Cano (coord.). Historia de la lengua española, 973-1009. Barcelona: Ariel.

Moya Corral, Juan Antonio. 2012. Perdido. Resultados de la terminación -ido en el corpus PRESEEA-Granada. Revista de Filología 111-130.

Moya Corral, Juan Antonio, Emilio J. García Wiedemann, Esteban T. Montoro, Marcin Sosinsky, María Concepción Torres, Francisca Pose Furest y Elisabeth Melguizo. 2012. La/d/ intervocálica en Granada: factores lingüísticos y sociales. Español hablado. Estudios sobre el corpus PRESEEA-Granada, 95-148. Varsovia: Instituto de Estudios Ibéricos e Iberoamericanos.

Moya Corral, Juan Antonio y Emilio García Wiedemann. 2009. La elisión de /d/ intervocálica en el español de Granada: factores lingüísticos. Pragmalingüística 17: 92-123.

Navarro, Manuel. 1995. El español hablado en Puerto Cabello. Valencia: Ediciones de la Universidad de Carabobo. Facultad de Ciencias de la Educación.

1983. La variación del segmento /d/ en Puerto Cabello. Boletín de la Academia Puertorriqueña de la Lengua Española 9: 65-72.

Penny, Ralph. 2000. Variación y cambio en español. Madrid: Gredos.

Perdomo, Lorena. 2015. Variaciones del fonema /d/ en contexto intervocálico: Un análisis sociolingüístico del habla caraqueña. Tesis de grado para optar al título de Antropóloga. Caracas: Universidad Central de Venezuela.

Samper Padilla, José Antonio, Clara E. Hernández, Magnolia Troya, Ana Ma Pérez Martín, y Marta SAmper Hernández. 2010. Un estudio de variación fonética en el marco del PRESEEA: realizaciones de /d/ entre los hablantes de nivel universitario de Las Palmas de Gran Canaria. Lingüística Española Actual 32: 5-34.

2011a. Elisión de /d/ en los niveles de estudios primario y universitario de Las Palmas de Gran Canaria. Comparación con otras comunidades de habla. En José Joaquín Bustos Tovar, Rafael Cano, E. Méndez y A. López (coords.), Sintaxis y análisis del discurso hablado en español. Homenaje a Antonio Narbona, vol. I, pp. 409-424. Sevilla: Universidad de Sevilla.

2011b. Estudio sociolingüístico de la variación de -/d/- en la ciudad de Las Palmas de Gran Canaria. Comunicación presentada en el XVI Congreso Internacional de la ALFAL, Alcalá de Henares. 
Sankoff, David, Sali Tagliamonte, Eric Smith. 2005. [En línea]. GoldVarb X: A variable rule application for Macintosh and Windows. Toronto: Universidad de Toronto. Disponible en http://individual.utoronto.ca/tagliamonte/goldvarb.html [Consulta: 15 abril 2014].

Silva-Corvalán, Carmen. 2001. Sociolingüistica y pragmática del español. Washington, D.C.: Georgetown University Press

Villena Ponsoda, Juan Andrés; Francisco Díaz Montesinos, Antonio M. Ávila Muñoz y M. ${ }^{a}$ Cruz Lasarte Cervantes, 2011. Interacción de factores fonéticos y gramaticales en la variación fonológica: la elisión de /d/ intervocálica en la variedad de los hablantes universitarios en la ciudad de Málaga. En Yolanda Congosto y Elena Méndez (eds.). Variación lingüistica y contacto de lenguas en el mundo hispánico. In memoriam Manuel Alvar, 311-359. Madrid/Frankfurt: Iberoamericana/Vervuert. 